\title{
Vinhaça de alambique e nitrogênio na cana-de-açúcar, em ambiente irrigado e não irrigado ${ }^{1}$
}

\author{
Ednaldo L. de Oliveira ${ }^{2}$, Luiz A. de B. Andrade ${ }^{3}$, Manoel A. de Faria ${ }^{4} \&$ Telde N. Custódio ${ }^{3}$
}

\begin{abstract}
RESUMO
Com o objetivo de estudar os efeitos da aplicação de vinhaça de alambique com e sem complementação nitrogenada na produtividade e qualidade tecnológica da cana-de-açúcar, em ambiente irrigado e não irrigado, conduziu-se este trabaIho em área do Alambique João Mendes (JM), localizado no município de Perdões, região sul do Estado de Minas Gerais. Para cada condição (com irrigação e sem irrigação) se estudaram os seguintes tratamentos: $\mathrm{T} 1\left(0 \mathrm{~m}^{3}\right.$ ha-1 de vinhaça + $0 \mathrm{~kg} \mathrm{ha}^{-1}$ de N); T2 (100 $\mathrm{m}^{3} \mathrm{ha}^{-1}$ de vinhaça $\left.+0 \mathrm{~kg} \mathrm{ha}^{-1} \mathrm{de} \mathrm{N}\right)$; T3 $\left(150 \mathrm{~m}^{3} \mathrm{ha}^{-1}\right.$ de vinhaça $+0 \mathrm{~kg} \mathrm{ha}^{-1}$ de N); T4 $\left(200 \mathrm{~m}^{3}\right.$ ha $^{-1}$ de vinhaça $+0 \mathrm{~kg} \mathrm{ha}^{-1}$ de N); T5 $\left(0 \mathrm{~m}^{3} \mathrm{ha}^{-1}\right.$ de vinhaça $\left.+60 \mathrm{~kg} \mathrm{ha}^{-1} \mathrm{de} \mathrm{N}\right)$; T6 $\left(100 \mathrm{~m}^{3} \mathrm{ha}^{-1}\right.$ de vinhaça + $60 \mathrm{~kg} \mathrm{ha}^{-1}$ de N); T7 (150 m ha-1 de vinhaça $+60 \mathrm{~kg} \mathrm{ha}^{-1}$ de N) e T8 $\left(200 \mathrm{~m}^{3} \mathrm{ha}^{-1}\right.$ de vinhaça $+60 \mathrm{~kg} \mathrm{ha}^{-1}$ de N). No ambiente irrigado o rendimento médio de colmos foi $15,25 \%$ maior que o obtido no ambiente não irrigado. A aplicação de $150 \mathrm{~m}^{3}$ ha-1 de vinhaça de alambique complementada com $60 \mathrm{~kg} \mathrm{ha}^{-1}$ de $\mathrm{N}$, foi o tratamento que propiciou o melhor resultado, exceto para teor de fibra (\%) cana.
\end{abstract}

Palavras-chaves: nitrogênio, cana-soca, irrigação, sequeiro

\section{Alembic vinasse and nitrogen in sugar cane in irrigated and non-irrigated areas}

\begin{abstract}
With the purpose of studying the effects of the application of alembic vinasse, both with and without nitrogen complementation on the yield and technological quality of sugar cane in irrigated and non-irrigated area, this work was conducted in the area of João Mendes Alembic (JM), situated in the town of Perdões, southern region of the State of Minas Gerais. Under each condition (with and without irrigation), the following treatments were studied: T1 $\left(0 \mathrm{~m}^{3} \mathrm{ha}^{-1}\right.$ de vinasse $+0 \mathrm{~kg} \mathrm{ha}^{-1}$ de N); T2 $\left(100 \mathrm{~m}^{3} \mathrm{ha}^{-1}\right.$ de vinasse $+0 \mathrm{~kg} \mathrm{ha}^{-1}$ de N); T3 $\left(150 \mathrm{~m}^{3} \mathrm{ha}^{-1}\right.$ de vinasse $+0 \mathrm{~kg} \mathrm{ha}^{-1} \mathrm{de}$ $\mathrm{N})$; T4 $\left(200 \mathrm{~m}^{3}\right.$ ha-1 de vinasse $\left.+0 \mathrm{~kg} \mathrm{ha}^{-1} \mathrm{de} \mathrm{N}\right)$; T5 $\left(0 \mathrm{~m}^{3} \mathrm{ha}^{-1}\right.$ de vinasse $\left.+60 \mathrm{~kg} \mathrm{ha}^{-1} \mathrm{de} \mathrm{N}\right)$; T6 $\left(100 \mathrm{~m}^{3} \mathrm{ha}^{-1}\right.$ de vinasse + $60 \mathrm{~kg} \mathrm{ha}^{-1}$ de N); T7 (150 m ha $\mathrm{ha}^{-1}$ de vinasse $+60 \mathrm{~kg} \mathrm{ha}^{-1}$ de N) e T8 $\left(200 \mathrm{~m}^{3} \mathrm{ha}^{-1}\right.$ de vinasse $\left.+60 \mathrm{~kg} \mathrm{ha}^{-1} \mathrm{de} \mathrm{N}\right)$. Under the irrigated conditions, the average yield of the stems was $15.25 \%$ greater than the one obtained in the nonirrigated area. The application of $150 \mathrm{~m}^{3} \mathrm{ha}^{-1}$ of alembic vinasse complemented by $60 \mathrm{~kg} \mathrm{ha}^{-1}$ of $\mathrm{N}$ was the treatment which provided the best result, except for the content of cane fiber (\%).
\end{abstract}

Key words: nitrogen, ratoon cane, irrigation, upland

\footnotetext{
1 Projeto financiado pela FAPEMIG (EDT 2730/06)

2 Instituto Federal do Norte de Minas Gerais - IFNMG/Campus Araçuai, MG. CP 97, CEP 39480-000, Januária, MG. Fone: (33) 3731-4448. E-mail: ednaldoliberato@yahoo.com.br

3 DAG/UFLA, Campus Universitário, C.P. 3037, CEP 37200-000, Lavras, MG. E-mail: laba@ufla.br; telde@ufla.br

${ }^{4}$ DEG/UFLA. E-mail: mafaria@ufla.br
} 


\section{INTRODUÇÃO}

De acordo com SEBRAE (2001) há em Minas Gerais cerca de 8.500 alambiques produzindo aproximadamente 200 milhões de litros de cachaça por safra. Associada à produção de cachaça artesanal se encontra a produção de vinhaça ou vinhoto, resíduo que sobra no alambique após a destilação, cujo líquido, devido a uma demanda biológica de oxigênio elevada, é um dos mais agressivos agentes poluidores e não deve ser despejado nos rios, lagos e lagoas. Segundo Franco et al. (2008), a aplicação de vinhaça na cultura da cana-de-açúcar pode substituir a adubação mineral, tanto para cana-planta quanto para cana-soca, a não ser que o produto seja aplicado em dosagens excessivas, podendo então diminuir os rendimentos e aumentar a salinidade dos solos.

Dentre as várias dosagens recomendadas para aplicação da vinhaça no solo como fertilizante, deve-se estar atento a composição da vinhaça, uma vez que esta é bastante variável e depende de uma série de fatores tais como: natureza e composição da matéria-prima utilizada, composição do mostro, tipo de fermentação, processo de destilação empregado, (Gava et al., 2001; Azania et al., 2003).

Paulino et al. (2002), trabalhando com produções agrícola e industrial de cana-de-açúcar submetida a doses de vinhaça, utilizando dados referentes às $4^{\text {a }}$ e $5^{\text {a }}$ colheitas $\left(3^{\mathrm{a}} \mathrm{e}\right.$ $4^{\mathrm{a}}$ socas). Concluíram que as doses intermediárias de vinhaça (300 e $450 \mathrm{~m}^{3} \mathrm{ha}^{-1}$ ) geraram os melhores índices de produtividades agrícola e industrial.

Os estudos de Andrade et al. (2000), objetivando avaliar os efeitos de fontes e doses de nitrogênio em soqueira de cana-de-açúcar, demonstraram que o rendimento de colmos e açúcar teórico recuperável além do brix, pol, pureza, açúcares redutores e fibra \% cana, não sofreram efeitos segnificativos, independente da fonte ou dose utilizada.

Quanto à irrigação em cana-de-açúcar, Guazzelli \& Paes (1997) obtiveram aumento de produtividade de até $30 \mathrm{t} \mathrm{ha}^{-1}$, correspondente a um acréscimo de $20,8 \%$ quanto à cana não irrigada. Dantas Neto et al. (2006), trabalhando com níveis de irrigação e adubação de cobertura em cana-soca, variedade SP79-1011, na zona da mata paraibana, concluíram que a irrigação e a adubação de cobertura atuaram de forma independente sobre as variáveis analisadas e que não houve efeito significativo de lâminas de irrigação na produtividade agrícola.

Fernandes (2005), avaliando a produtividade de cana-deaçúcar sob irrigação por aspersão para produção de cachaça artesanal na região de Salinas, Norte de Minas, obteve rendimento médio de $242 \mathrm{t} \mathrm{ha}^{-1}$ em cana planta.

Não há, até o momento, informações seguras relativas a resultados experimentais e recomendações sobre o uso de vinhaça obtida de alambiques. As inferências atuais se baseiam no uso da vinhaça produzida em destilarias além do que, para fertilização de soqueiras no sistema de cana colhida crua, existem dúvidas quanto a real necessidade da fertilização nitrogenada em áreas com e sem aplicação de vinhaça.

Propôs-se, no trabalho, estudar os efeitos da aplicação de vinhaça de alambique com e sem complementação nitrogenada, na produtividade e qualidade tecnológica da cana-de- açúcar, em ambiente irrigado e não irrigado, na região sul de Minas Gerais.

\section{MATERIAL E MÉTODOS}

O experimento foi conduzido em uma área de aproximadamente 1,0 ha, cultivada com cana ( $2^{\mathrm{a}}$ soca) no alambique João Mendes (Alambique JM), município de Perdões, situado na região sul do Estado de Minas Gerais, cujas coordenadas geográficas são $21^{\circ} 05^{\prime}$ de latitude sul; 45 05’ de longitude oeste e altitude média de $826 \mathrm{~m}$.

O clima da região, segundo a classificação de Köppen, é do tipo Cwb, caracterizado por uma estação seca entre abril e setembro e uma estação chuvosa de outubro a março. As evaporações médias do tanque Classe A (ECA) e os índices pluviométricos mensais referentes ao período de condução do experimento, são apresentados na Figura 1.

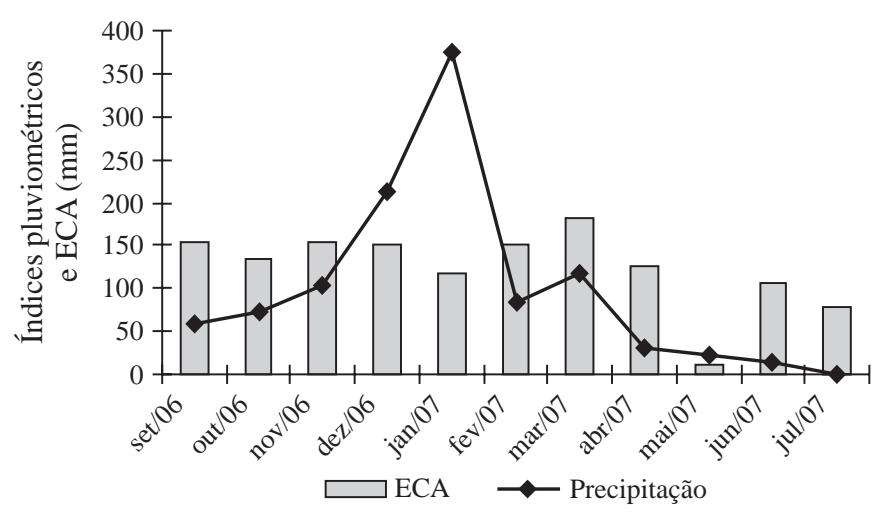

Figura 1. Variação mensal da precipitação e ECA dos anos 2006 e 2007, no período estudado

Amostras de solo foram retiradas em setembro de 2006, nas camadas de 0-20 e 20-40 cm, para determinação das características químicas cujos resultados estão apresentados na Tabela 1. O solo no qual se instalou o experimento, caracteriza-se como Latossolo Vermelho Amarelo distrófico (EMBRAPA, 2006), com relevo plano a suave ondulado.

A variedade de cana-de-açúcar utilizada foi a SP 80-1816. Em Minas Gerais, nas principais regiões produtoras de cachaça, esta cultivar é considerada de alta produtividade agrícola, alto teor de sacarose, maturação média, médio número de colmos por metro linear, média exigência em fertilidade do solo, não floresce e é tolerante às principais doenças (Silveira et al., 2002).

Para cada ambiente (irrigado e não irrigado) utilizou-se, o delineamento em blocos casualizados, com três repetições, em esquema fatorial $2 \times 4$, sendo duas doses de adubação nitrogenada ( 0 e $60 \mathrm{~kg} \mathrm{ha}^{-1}$ de $\mathrm{N}$ ) e quatro dosagens de vinhaça de alambique $\left(0,100,150\right.$ e $\left.200 \mathrm{~m}^{3} \mathrm{ha}^{-1}\right)$.

Os tratamentos foram: T1 $\left(0 \mathrm{~m}^{3} \mathrm{ha}^{-1}\right.$ de vinhaça + $0 \mathrm{~kg} \mathrm{ha}^{-1}$ de $\left.\mathrm{N}\right)$; T2 (100 $\mathrm{m}^{3} \mathrm{ha}^{-1}$ de vinhaça $+0 \mathrm{~kg} \mathrm{ha}^{-1}$ de $\mathrm{N})$; T3 (150 $\mathrm{m}^{3} \mathrm{ha}^{-1}$ de vinhaça $+0 \mathrm{~kg} \mathrm{ha}^{-1}$ de N); T4 (200 $\mathrm{m}^{3}$ ha $^{-1}$ de vinhaça $+0 \mathrm{~kg} \mathrm{ha}^{-1}$ de $\left.\mathrm{N}\right)$; T5 $\left(0 \mathrm{~m}^{3} \mathrm{ha}^{-1}\right.$ de vinhaça $+60 \mathrm{~kg} \mathrm{ha}^{-1}$ de $\left.\mathrm{N}\right)$; T6 $\left(100 \mathrm{~m}^{3} \mathrm{ha}^{-1}\right.$ de vinhaça + 
Tabela 1. Resultado das análises químicas das amostras do solo da área experimental

\begin{tabular}{|c|c|c|c|c|}
\hline \multirow{2}{*}{ Sigla } & \multirow{2}{*}{ Descrição } & \multirow{2}{*}{ Unidade } & \multicolumn{2}{|c|}{ Camadas (cm) } \\
\hline & & & $0-20$ & $20-40$ \\
\hline pH & Em água $(1: 2,5)$ & - & 5,2 & 5,5 \\
\hline$P$ & Fósforo disponível & $\mathrm{mg} \mathrm{dm}-3$ & 0,9 & 0,6 \\
\hline K & Potássio disponível & $\mathrm{mg} \mathrm{dm}^{-3}$ & 23 & 17 \\
\hline $\mathrm{Ca}^{2+}$ & Cálcio trocável & $\mathrm{cmol}_{\mathrm{c}} \mathrm{dm}^{-3}$ & 1,0 & 1,1 \\
\hline $\mathrm{Mg}^{2+}$ & Magnésio trocável & $\mathrm{cmol}_{\mathrm{c}} \mathrm{dm}^{-3}$ & 0,4 & 0,6 \\
\hline $\mathrm{Al}^{3+}$ & Acidez trocável & $\mathrm{cmol}_{\mathrm{c}} \mathrm{dm}^{-3}$ & 0,2 & 0,2 \\
\hline $\mathrm{H}+\mathrm{Al}$ & Acidez potencial & $\mathrm{cmol}_{\mathrm{c}} \mathrm{dm}^{-3}$ & 3,2 & 3,2 \\
\hline MO & Matéria orgânica & dag $\mathrm{kg}^{-1}$ & 2,0 & 1,9 \\
\hline $\mathrm{Zn}$ & Zinco disponível & $\mathrm{mg} \mathrm{dm}^{-3}$ & 0,2 & 0,8 \\
\hline $\mathrm{Fe}$ & Ferro disponível & $\mathrm{mg} \mathrm{dm}^{-3}$ & 45,6 & 43,5 \\
\hline $\mathrm{Mn}$ & Manganês disponível & $\mathrm{mg} \mathrm{dm}^{-3}$ & 2,7 & 3,0 \\
\hline $\mathrm{Cu}$ & Cobre disponível & $\mathrm{mg} \mathrm{dm}^{-3}$ & 20,2 & 8,4 \\
\hline B & Boro disponível & $\mathrm{mg} \mathrm{dm}^{-3}$ & 0,2 & 0,2 \\
\hline S & Enxofre & $\mathrm{mg} \mathrm{dm}^{-3}$ & 14,3 & 7,5 \\
\hline
\end{tabular}

Análises realizadas pelo Laboratório de Fertilidade do Solo - DCS/UFLA.P e K extrator Mehlich 1 ; $\mathrm{Ca}, \mathrm{Mg}$ e Al: extrator $\mathrm{KCl} 1 \mathrm{~N} ; \mathrm{H}+$ Al extraídos com acetato de cálcio $1 \mathrm{~N}$, pH 7,0

$60 \mathrm{~kg} \mathrm{ha}^{-1}$ de N); T7 (150 m ha-1 de vinhaça $+60 \mathrm{~kg} \mathrm{ha}^{-1}$ de N) e T8 (200 $\mathrm{m}^{3} \mathrm{ha}^{-1}$ de vinhaça $+60 \mathrm{~kg} \mathrm{ha}^{-1}$ de N), aplicados em dosagem única e de forma manual, sobre o palhiço remanescente da cana-soca, em que as doses de vinhaças foram aplicadas com o auxílio de um tanque tracionado a trator; posteriormente, o nitrogênio foi aplicado em cobertura nos tratamentos correspondentes, tanto na área irrigada como na não irrigada.

As parcelas se constituíram de cinco linhas de cana-deaçúcar com espaçamento de 1,40 m entre linhas e comprimento de 14,30 m; a área útil de cada parcela correspondeu às três linhas centrais, perfazendo $60,48 \mathrm{~m}^{2}$, enquanto as parcelas foram delimitadas por estacas de madeira e separadas por carreadores transversais de 1,00 m de largura.

Para a irrigação utilizaram-se aspersores de média pressão (300 kPa), com dois bocais (14 x $5 \mathrm{~mm})$, instalados no espaçamento 30 x $24 \mathrm{~m}$, precipitação do aspersor 7,3 $\mathrm{mm} \mathrm{h}^{-1}$, com funcionamento setorial e se adotou um fator de disponibilidade de água no solo de 0,65; a evapotranspiração potencial de referência foi calculada com base na evaporação do tanque Classe A, conforme Costa et al. (2007). Para o cálculo da evapotranspiração da cultura foram adotados os valores de coeficientes da cultura utilizados por Moura et al. (2005). Para o cálculo do armazenamento de água no solo se determinaram as características físico-hídricas, por meio de amostragem de solo, nas camadas de 0-20 e 20-40 cm (Tabela 2). Adotou-se, como capacidade de campo do solo, a umidade correspondente ao potencial mátrico de $-10 \mathrm{kPa}$.

A primeira irrigação foi realizada em 13/09/2007, elevando-se a umidade do solo a capacidade de campo, na camada de 0 a $40 \mathrm{~cm}$; a partir daí realizou-se o manejo da irrigação com turno de rega fixo de 7 dias e se cortou a irrigação aos 45 dias antes do início da colheita do ensaio.

A composição química média da vinhaça utilizada no experimento está apresentada na Tabela 3.

A colheita do experimento foi realizada no período de 09 a 16/07/2007; obteve-se o rendimento de colmos por meio
Tabela 2. Características* físico-hídricas do solo da área experimental

\begin{tabular}{lcc}
\hline \multirow{2}{*}{ Variável } & \multicolumn{2}{c}{ Camadas (cm) } \\
\cline { 2 - 3 } Granulometria (\%) & $\mathbf{0 - 2 0}$ & $\mathbf{2 0 - 4 0}$ \\
Areia & 41 & 42 \\
Silte & 16 & 15 \\
Argila & 43 & 43 \\
Casse textural & Argilosa & Argilosa \\
C.C. & 27,41 & 25,61 \\
PMP & 35,98 & 42,74 \\
dg $\left(\mathrm{g} \mathrm{cm}\right.$ c $\left.^{-3}\right)$ & 1,10 & 1,15 \\
${ }^{*} D g-$ densidade global; PPM - ponto de murcha permanente; C.C. - capacidade de campo. 0s \\
valores de C.C. e PPM foram determinadas com base em peso
\end{tabular}

Tabela 3. Composição química média da vinhaça utilizada no experimento, em 2006

\begin{tabular}{cc}
\hline Variável & Resultado $\left(\mathbf{k g ~ m}^{-3}\right)$ \\
Carbono & 9,4 \\
Nitrogênio & 0,23 \\
$\mathrm{P}_{2} \mathrm{O}_{5}$ & 0,071 \\
$\mathrm{~K}_{2} \mathrm{O}$ & 0,73 \\
$\mathrm{CaO}$ & 0,14 \\
$\mathrm{MgO}$ & 0,33 \\
\hline
\end{tabular}

Fonte: Laboratório de Análise Química de Solo e Planta/UFScar

de pesagens em balança tipo dinamômetro, com capacidade para $120 \mathrm{~kg}$, seguindo-se metodologia usada por Leite et al. (2008); posteriormente, realizou-se a transformação do peso de colmos da área útil da parcela em toneladas de colmos por hectare.

Para avaliar as características tecnológicas da cana imediatamente após a colheita foram retirados, aleatoriamente, 12 colmos seguidos na área útil de cada parcela, eliminando-se o palmito e a palha; após a identificação, os colmos foram enfeixados, identificados e enviados ao Laboratório da Destilaria Alvorada do Bebedouro Ltda., em Guaranésia, Minas Gerais, para determinação de sólidos solúveis (Brix em \%), sacarose (Pol do caldo extraído \%), fibra industrial (Fibra \%), pureza do caldo extraído (Pureza \%), açúcares redutores (AR \%) e açúcar total recuperável (ATR). Entende-se como Brix a quantidade total de sólidos solúveis (sacarose, frutose e glicose), como pol a porcentagem de sacarose aparente nos sólidos solúveis e, como fibra, os constituintes insolúveis; já pureza da cana corresponde à relação (Pol/Brix) x 100; os açúcares redutores se referem à soma de glicose + frutose.

Os dados foram submetidos à análise de variância, com realização do teste de F, sendo as comparações entre os tratamentos integrantes do esquema fatorial efetuada por meio de análises de regressão (Yassin et al., 2002).

\section{RESULTADOS E DISCUSSÃO}

O resumo da análise de variância para rendimento de colmos da cana-de-açúcar na colheita em função das diferentes doses de vinhaça e nitrogênio aplicadas, nos dois ambientes (irrigação e não irrigado), é apresentado na Tabela 4. 
Tabela 4. Resumo da análise de variância para o rendimento de colmos em função dos tratamentos aplicados

\begin{tabular}{lcrrc}
\hline Fonte de variação & G.L. & SQ & QM & Fc \\
Bloco & 2 & 298,35 & 149,17 & $0,91^{\text {ns }}$ \\
Ambientes & 1 & 1511,67 & 1511,67 & $9,218^{\text {** }}$ \\
Tratamentos & 7 & 4192,44 & 598,92 & $3,652^{\text {** }}$ \\
Ambientes x tratamentos & 7 & 708,37 & 101,19 & $0,61^{\text {ns }}$ \\
\hline \multicolumn{1}{c}{ CV (\%) } & \multicolumn{5}{c}{16,16} \\
\hline
\end{tabular}

** Significativo a nível de $1 \%$ de probabilidade pelo teste $\mathrm{F}$; ns não significativo

Verifica-se, em relação à produtividade de colmos, a ocorrência de efeitos significativos para ambientes e tratamentos. No ambiente irrigado a produtividade média obtida $\left(84,85\right.$ t ha $\left.^{-1}\right)$ foi significativamente maior que aquela obtida no ambiente não irrigado (73,62 t ha $\left.{ }^{-1}\right)$, conforme Tabela 5.

Tabela 5. Valores médios obtidos para rendimento de colmos (t ha-1) em ambiente irrigado e não irrigado

\begin{tabular}{cc}
\hline Irrigação & Rendimento de colmos (t ha-1) \\
Com & $84,85 \mathrm{a}$ \\
Sem & $73,62 \mathrm{~b}$ \\
\hline
\end{tabular}

No sentido das colunas médias seguidas de letras diferentes são estatisticamente diferentes

O aumento médio de produtividade, obtido no presente trabalho com a cana irrigada em relação à não irrigada, foi de 15,25\%; já Guazzelli \& Paes (1997) obtiveram aumento de produtividade da ordem de 20,8\%.

O baixo percentual no aumento de produtividade encontrado entre a área irrigada e não irrigada neste trabalho se deve, provavelmente, ao fato de que, nos quatro primeiros meses após a brotação da cana-soca (11 de setembro de 2006 a 31 de janeiro de 2007), conforme Tabela 6, a precipitação, ocorrida no período pode ter sido suficiente para evitar prejuízos à brotação inicial refletindo no rendimento final da cultura,

Segundo Dalri \& Cruz (2008) a resposta da cana de açúcar à irrigação durante o período vegetativo é maior que durante a última parte do período de formação da colheita, quando a área foliar ativa está diminuindo e a cultura apre-

Tabela 6. Dados médios de precipitação, estimativa da evapotranspiração acumulada e déficit hídrico, durante o período estudado

\begin{tabular}{lccc}
\hline \multicolumn{1}{c}{ Período } & Precipitação & Etcacumulada & Défict hídrico \\
\cline { 2 - 4 } Setembro/2006 & \multicolumn{3}{c}{$\mathbf{( m m )}$} \\
Outubro/2006 & 58,44 & 25,36 & \\
Novembro/2006 & 71,34 & 61,28 & \\
Dezembro/2006 & 102,31 & 101,40 & \\
Janeiro/2007 & 212,29 & 107,14 & \\
Fevereiro/2007 & 375,54 & 90,60 & \\
Março/2007 & 84,72 & 119,41 & $-34,69$ \\
Abril/2007 & 116,38 & 125,47 & $-90,09$ \\
Maio/2007 & 29,86 & 104,33 & $-74,47$ \\
Junho/2007 & 23,33 & 65,98 & $-42,65$ \\
Julho/2007 & 13,15 & 86,20 & $-73,05$ \\
\hline \multicolumn{1}{c}{ Total } & 0,0 & 40,95 & $-40,95$ \\
\hline
\end{tabular}

senta menor capacidade de resposta à luz solar.

Apresentam-se, na Tabela 7, os resultados médios obtidos (ambiente irrigado e não irrigado) para rendimento de colmos, em função dos tratamentos aplicados.

Verifica-se, pela Tabela 7, que o melhor tratamento cor-

Tabela 7. Valores médios obtidos (ambiente irrigado e não irrigado) para rendimento de colmos (t ha-1) em função dos tratamentos aplicados

\begin{tabular}{ll}
\hline \multicolumn{1}{c}{ Tratamentos } & $\begin{array}{c}\text { Rendimento de Colmos } \\
(\mathbf{t} \text { ha-1) }\end{array}$ \\
Vinhaça $=150 \mathrm{~m}^{3} \mathrm{ha}^{-1}+$ Nitrogênio $=60 \mathrm{~kg} \mathrm{ha}^{-1}$ & $95,08 \mathrm{a}$ \\
Vinhaça $=100 \mathrm{~m}^{3} \mathrm{ha}^{-1}+$ Nitrogênio $=60 \mathrm{~kg} \mathrm{ha}^{-1}$ & $84,35 \mathrm{ab}$ \\
Vinhaça $=200 \mathrm{~m}^{3} \mathrm{ha}^{-1}+$ Nitrogênio $=6 \mathrm{~kg} \mathrm{ha}^{-1}$ & $83,69 \mathrm{ab}$ \\
Vinhaça $=100 \mathrm{~m}^{3} \mathrm{ha}^{-1}+$ Nitrogênio $=0 \mathrm{~kg} \mathrm{ha}^{-1}$ & $83,54 \mathrm{ab}$ \\
Vinhaça $=2000 \mathrm{~m}^{3} \mathrm{ha}^{-1}+$ Nitrogênio $=60 \mathrm{~kg} \mathrm{ha}^{-1}$ & $81,55 \mathrm{ab}$ \\
Vinhaça $=150 \mathrm{~m}^{3} \mathrm{ha}^{-1}+$ Nitrogênio $=0 \mathrm{~kg} \mathrm{ha}^{-1}$ & $71,45 \mathrm{ab}$ \\
Vinhaça $=0 \mathrm{~m}^{3} \mathrm{ha}^{-1}+$ Nitrogênio $=0 \mathrm{~kg} \mathrm{ha}^{-1}$ & $70,46 \mathrm{~b}$ \\
Vinhaça $=0 \mathrm{~m}^{3} \mathrm{ha}^{-1}+$ Nitrogênio $=60 \mathrm{~kg} \mathrm{ha}^{-1}$ & $63,73 \mathrm{~b}$ \\
\hline
\end{tabular}

No sentido das colunas médias seguidas de letras diferentes são estatisticamente diferentes

respondeu à aplicação de $150 \mathrm{~m}^{3} \mathrm{ha}^{-1}$ de vinhaça, complementada com $60 \mathrm{~kg}$ de $\mathrm{N} \mathrm{ha}^{-1}$, com um rendimento médio de 95,08 $\mathrm{tha}^{-1}$, enquanto os piores tratamentos corresponderam à não aplicação de vinhaça, complementada ou não com nitrogênio, com rendimentos médios de 63,73 e 7 0,47 t ha-1, respectivamente.

Robaina et al. (1984) concluíram, estudando o comportamento de produtividade de cana-soca em função da aplicação de vinhaça de destilaria complementada com nitrogênio, que a aplicação de vinhaça proporcionou acréscimos significativos na produtividade de cana-soca, sendo que a complementação com nitrogênio não apresentou respostas diferenciadas entre si. Por sua vez, Penatti et al. (2001), trabalhando com doses crescentes de 0, 50, 100 e $150 \mathrm{~m}^{3} \mathrm{ha}^{-1}$ de vinhaça de destilaria complementada com 0, 50, 100 e $150 \mathrm{~kg} \mathrm{ha}^{-1}$ de nitrogênio, combinando doses de vinhaça com doses de nitrogênio, encontraram efeito linear e significativo da vinhaça sobre a produção de cana-de-açúcar; eles, concluíram, também, que nas doses de 50 e $100 \mathrm{~m}^{3} \mathrm{ha}^{-1}$ de vinhaça a produtividade de cana-de-açúcar respondeu linear e significativamente à complementação nitrogenada.

Figueiredo et al. (2004) concluiu, estudando os efeitos da vinhaça e de sua complementação nitrogenada, que a aplicação da vinhaça, complementada ou não com nitrogênio, aumentou os rendimentos de colmos; já Quintela et al. (2002) afirmam que o rendimento da variedade SP-1842 não foi afetado pela aplicação de vinhaça na dosagem de $100 \mathrm{~m}^{3} \mathrm{ha}^{-1}$ em área não irrigada.

\section{Características tecnológicas da cana-de-açúcar}

O resumo das análises de variância para as características tecnológicas da cana-de-açúcar, em função dos tratamentos aplicados, está apresentado na Tabela 8.

Analisando-se a Tabela 8 , verifica-se que não houve efeito significativo de ambiente e da interação ambientes $\mathrm{x}$ tratamentos para nenhuma das características tecnológicas estudadas; verificou-se apenas efeito significativo de 
Tabela 8. Resumo da análise de variância para as características tecnológicas da cana de açúcar, variedade SP80-1816, em função dos tratamentos aplicados

\begin{tabular}{lccccccc}
\hline \multirow{2}{*}{ Fonte de Variação } & \multirow{2}{*}{ GL. } & \multicolumn{7}{c}{ Q.M. } \\
\cline { 3 - 8 } & & Brix & AR & ATR & Fibra & Pol & Pureza \\
Bloco & 3 & 0,22 & 0,004 & 5,47 & 0,13 & 0,07 & 4,36 \\
Ambientes & 1 & 1,43 & 0,006 & 51,00 & 0,09 & 1,11 & 8,23 \\
Tratamentos & 7 & 0,63 & 0,015 & 51,56 & $0,31^{*}$ & 0,55 & 1,78 \\
Ambientes x tratamentos & 7 & 0,56 & 0,002 & 64,74 & 0,24 & 0,72 & 2,75 \\
C.V (\%) & & 3,55 & 25,93 & 3,99 & 3,4 & 4,34 & 1,99 \\
\hline
\end{tabular}

* Significativo a nível de $5 \%$ de probabilidade pelo teste $\mathrm{F}$

tratamentos para a característica fibra (\%) cana.

Na Tabela 9 se apresentam os resultados médios obtidos (ambiente irrigado e não irrigado) para a característica fibra (\%) cana, em função dos tratamentos aplicados.

Verifica-se, pela Tabela 9, que o menor valor médio para fibra foi observado no tratamento correspondente à aplicação de $200 \mathrm{~m}^{3} \mathrm{ha}^{-1}$ de vinhaça, complementada com $60 \mathrm{~kg}$ de $\mathrm{N} \mathrm{ha}^{-1}(10,33 \%)$, enquanto o maior valor foi constatado no tratamento referente à aplicação de $0 \mathrm{~m}^{3}$ de vinhaça $+0 \mathrm{~kg}$ de $\mathrm{N} \mathrm{ha}^{-1}(11,33 \%)$.

Tabela 9. Valores médios obtidos (ambiente irrigado e não irrigado) para fibra (\%) cana, em função dos tratamentos aplicados

\begin{tabular}{cc}
\hline \multicolumn{1}{c}{ Tratamentos } & $\begin{array}{c}\text { Fibra (\%) cana } \\
\text { Média }\end{array}$ \\
Vinhaça $=200 \mathrm{~m}^{3} \mathrm{ha}^{-1}+$ Nitrogênio $=60 \mathrm{~kg} \mathrm{ha}^{-1}$ & $10,33 \mathrm{a}$ \\
Vinhaça $=100 \mathrm{~m}^{3} \mathrm{ha}^{-1}+$ Nitrogênio $=0 \mathrm{~kg} \mathrm{ha}^{-1}$ & $11,00 \mathrm{ab}$ \\
Vinhaça $=150 \mathrm{~m}^{3} \mathrm{ha}^{-1}+$ Nitrogênio $=0 \mathrm{~kg} \mathrm{ha}^{-1}$ & $11,00 \mathrm{ab}$ \\
Vinhaça $=200 \mathrm{~m}^{3} \mathrm{ha}^{-1}+$ Nitrogênio $=0 \mathrm{~kg} \mathrm{ha}^{-1}$ & $11,00 \mathrm{ab}$ \\
Vinhaça $=0 \mathrm{~m}^{3} \mathrm{ha}^{-1}+$ Nitrogênio $=60 \mathrm{~kg} \mathrm{ha}^{-1}$ & $11,00 \mathrm{ab}$ \\
Vinhaça $=100 \mathrm{~m}^{3} \mathrm{ha}^{-1}+$ Nitrogênio $=60 \mathrm{~kg} \mathrm{ha}^{-1}$ & $11,00 \mathrm{ab}$ \\
Vinhaça $=150 \mathrm{~m}^{3} \mathrm{ha}^{-1}+$ Nitrogênio $=60 \mathrm{~kg} \mathrm{ha}^{-1}$ & $11,00 \mathrm{ab}$ \\
Vinhaça $=0 \mathrm{~m}^{3} \mathrm{ha}^{-1}+$ Nitrogênio $=0 \mathrm{~kg} \mathrm{ha}^{-1}$ & $11,33 \mathrm{~b}$ \\
\hline
\end{tabular}

No sentido das colunas, médias seguidas por letras diferentes são estatisticamente diferentes

Menor teor de fibra significa cana com menor dureza, que é interessante para os produtores de cachaça de alambique, haja vista que, devido às moendas utilizadas, renderá maior volume de caldo extraído e, como conseqüência, contribuirá para aumentar o rendimento final de cachaça; já para as destilarias, teores de fibra na cana inferiores a 10,5\% é uma característica indesejável em virtude do balanço energético (Horii, 2004). Segundo este autor, com menos fibra se tem que queimar maior quantidade de bagaço para se manter o poder calorífico das caldeiras.

As demais características estudadas não foram afetadas pelos tratamentos cujos valores médios estão apresentados na Tabela 10, sendo que esses valores estão na faixa aceitável da composição dos colmos de cana-de-açúcar usada como matéria-prima para o uso de açúcar, álcool e cachaça (Quintela et al., 2002; Farias et al., 2008; Leite et al., 2008).

Verificou-se que, de modo geral, as doses aplicadas de vinhaça e nitrogênio não afetaram a maturação da cana não influenciando, portanto, nos valores obtidos para ATR e demais características tecnológicas.
Tabela 10. Valores médios para as características tecnológicas de cana de açúcar em função dos tratamentos aplicados em ambiente irrigado e não irrigado

\begin{tabular}{cccccc}
\hline \multirow{2}{*}{ Tratamentos } & ATR $(\mathbf{k g})$ & Brix & \multicolumn{1}{c}{ Pol } & Pureza & AR \\
\cline { 4 - 6 } (t de cana)-1 & \multicolumn{4}{c}{ (\%) cana } \\
T1 & $167,00 \mathrm{a}$ & $18,88 \mathrm{a}$ & $17,00 \mathrm{a}$ & $92,84 \mathrm{a}$ & $0,39 \mathrm{a}$ \\
T2 & $165,64 \mathrm{a}$ & $18,66 \mathrm{a}$ & $16,86 \mathrm{a}$ & $92,92 \mathrm{a}$ & $0,39 \mathrm{a}$ \\
T3 & $167,12 \mathrm{a}$ & $18,70 \mathrm{a}$ & $17,18 \mathrm{a}$ & $93,58 \mathrm{a}$ & $0,37 \mathrm{a}$ \\
T4 & $165,52 \mathrm{a}$ & $18,50 \mathrm{a}$ & $16,96 \mathrm{a}$ & $92,89 \mathrm{a}$ & $0,26 \mathrm{a}$ \\
T5 & $165,66 \mathrm{a}$ & $18,73 \mathrm{a}$ & $16,86 \mathrm{a}$ & $92,02 \mathrm{a}$ & $0,41 \mathrm{a}$ \\
T6 & $161,25 \mathrm{a}$ & $18,08 \mathrm{a}$ & $16,46 \mathrm{a}$ & $92,50 \mathrm{a}$ & $0,41 \mathrm{a}$ \\
T7 & $158,82 \mathrm{a}$ & $17,95 \mathrm{a}$ & $16,26 \mathrm{a}$ & $92,02 \mathrm{a}$ & $0,41 \mathrm{a}$ \\
T8 & $164,13 \mathrm{a}$ & $18,53 \mathrm{a}$ & $16,94 \mathrm{a}$ & $92,20 \mathrm{a}$ & $0,42 \mathrm{a}$ \\
\hline
\end{tabular}

Letras iguais na mesma coluna não diferem significativamente entre si pelo teste Tukey

\section{CONCLUSÕES}

1. O uso da irrigação por aspersão proporcionou um rendimento médio de colmos $15,25 \%$, maior que o obtido no ambiente não irrigado.

2. A aplicação de $150 \mathrm{~m}^{3} \mathrm{ha}^{-1}$ de vinhaça de alambique complementada com $60 \mathrm{~kg}$ de $\mathrm{N} \mathrm{ha}^{-1}$ proporcionou o maior rendimento de colmos, não afetando as características tecnológicas da cana, exceto a fibra.

\section{AgRADECIMENTOS}

À Fundação de Amparo à Pesquisa do Estado de Minas Gerais - FAPEMIG, que fomentou este trabalho; a Cachaçaria João Mendes, que cedeu toda a estrutura necessária para a instalação e condução dos ensaios; enfim, aos revisores do trabalho, pela valiosa contribuição.

\section{LITERATURA CITADA}

Andrade, L. A. de B.; Bocardo, M. R.; Correa, J. B. D.; Carvalho, G. J. de. Efeitos do nitrogênio, aplicado nas formas fluída ou sólida, em soqueira de cana-de-açúcar. Ciência e Agrotecnologia, v.24, n.2, p.516-520, 2000.

Azania, A. A. P. M.; Marques, M. O.; Pavani, M. C. M. D.; Azania, C. A. M. Germinação de sementes de Sida rhombifolia e Brachiaria decumbens influenciada por vinhaça, flegmaça e óleo de fúsel. Planta Daninha, v.21, n.3, p.443-49, 2003.

Costa, E. L. da.; Macêdo, G. A. R.; Simão, F. R. Deniz, R. S. Irrigação da cana de açúcar. Informe Agropecuário, v.28, n.239, p.56-63, 2007.

Dalri, A. B.; Cruz, R. L. Produtividade da cana de açúcar fertirrigada com $\mathrm{N}$ e K via gotejamento subsuperficial. Engenharia Agrícola, v.28, n.3, p.516-524, 2008.

Dantas Neto, J.; Figueredo, J. L. da C.; Farias, C. H. de A.; Azevedo, H. M. de; Azevedo, C. A. V. de. Resposta da cana-deaçúcar, primeira soca, a níveis de irrigação e adubação de cobertura. Revista Brasileira de Engenharia Agrícola e Ambiental, v.10, n.2, p.283-288, 2006.

EMBRAPA - Empresa Brasileira de Pesquisa Agropecuária. Sistema brasileiro de classificação de solos. Rio de Janeiro: Embrapa Solos, 2006. 306p. 
Farias, C. H. A.; Fernandes, P. D.; Azevedo, H. M.; Dantas Neto, J. Índices de crescimento da cana de açúcar irrigada e de sequeiro no Estado da Paraíba. Revista Brasileira de Engenharia Agrícola e Ambiental, v.12, n.4, p.356-362, 2008.

Fernandes, O. W. B. Avaliação de variedades de cana-de-açúcar para produção de cachaça artesanal e a interferência dos resultados no comportamento do produtor na região de SalinasMG: Uma contribuição extensionista. Seropédica: UFRRJ, 2005. 69p. Dissertação Mestrado

Figueiredo, P. A. M.; Andrade, L. A. B. ; Barbosa, M. H. P. ; Quintela, A. C. R. ; Heinrichs, R. ; Correa, J. B. D . Consequências do palhiço, vinhaça e herbicidas no matocontrole em área de cana crua, variedade SP80-1842. STAB - Açúcar Álcool e Subprodutos, v.22, n.4, p.34-37, 2004.

Franco, A. ;Marques, M. O. ;Melo, W. J. de. Sugarcane grown in an oxisol amended with sewage sludge and vinasse: Nitrogen contents in soil and plant. Scientia Agrícola, v.65, n.4, p.408-414, 2008.

Gava, J. C.; Trivelin, P. C. O.; Oliveira, M. M.; Penatti, C. P. Crescimento e acúmulo de nitrogênio em cana-de-açúcar cultivada em solo coberto com palhada. Pesquisa Agropecuária Brasileira, v.36, n.11, p.1347-1354, 2001.

Guazzelli, M. A. N.; Paes, L. D. Irrigação de cana-de-açúcar comercial. In: seminário copersucar de tecnologia agronômica, 7, 1997. Piracicaba. Anais... Piracicaba: COPERSUCAR, 1997. 11p.

Horii, J. A cana-de-açúcar como matéria-prima. Piracicaba: ESALQ/USP, 2004. 27p.

Leite, G. M. V.; Andrade, L. A. B de.; Garcia, J. C.; Antônio dos Anjos, I. efeitos de fontes e doses de silicato de cálcio no rendimento agrícola e na qualidade tecnológica da cana-de-açúcar, cultivar sp80-1816. Ciência e Agrotecnologia, v.32, n.4, p.1120-1125, 2008.
Moura, M. V. P. da S. Farias, C. H. de A. V. Dantas Neto, J. Azevedo, H. M. de. Pordeus, R. V. Doses de adubação nitrogenada e potássica em cobertura na cultura da cana de açúcar, primeira soca, com e sem irrigação. Ciência e Agrotecnologia, v.29, n.4, p.753-760, 2005.

Paulino, A. F.; Medina, C. de C.; Robaina, C. R. P.; Laurani, R. A. Produções agrícola e industrial de cana-de-açúcar submetida a doses de vinhaça. Ciências Agrárias, v.23, n.2, p.145-150, 2002.

Penatti, C. P.; Araújo, J. V.; Forti, J. A.; Ribeiro, R. Doses de vinhaça e nitrogênio aplicadas em cana-soca durante quatro safras em solo LV - Usina São José da Estiva. STAB. Açúcar e Álcool e Subprodutos, v.19, n.5, p.38-41, 2001.

Quintela, A. C. R. ; Andrade, L. A. de B.; Corrêa, J. B. D.; Rezende, P. M. . Controle de plantas daninhas em cana crua (cultivar RB835089) no sistema integrado palhiço, herbicida e vinhaça. STAB - Açúcar Alcool e Subprodutos, v.20, n.4, p.38-42, 2002.

Robaina, A. A.; Vjieira, J. R.; Azevedo, D. F.; Bolsanello, J.; Manhães, M. S. dos. Doses e complementação mineral da vinhaça em socas de cana de açúcar. Brasil Açucareiro, v.102, n.1, p.26-33, 1984.

SEBRAE - Serviço Brasileiro de Apoio às Micro e Pequenas Empresas. Diagnóstico da cachaça de Minas Gerais. 1.ed. Belo Horizonte: SEBRAE, 2001. 259p.

Silveira, L. C. I. da; Barbosa, M. H. P.; Oliveira, M. W. de. Manejo de variedades de cana-de-açúcar predominantes nas principais regiões produtoras de cachaça de Minas Gerais. Informe Agropecuário, v.23, n.217, p.25-32, 2002.

Yassin, N.; Morais, A. R. de; Muniz, J. A. Análise de variância em um experimento fatorial de dois fatores com tratamentos adicionais. Ciência e Agrotecnologia, p.1541-1547, 2002. Edição especial. 